\section{Anti-VEGF therapy for choroidal neovascularisation previously treated with photodynamic therapy}

S Jyothi, HR Chowdhury, V Chong and

S Sivaprasad
Laser and Retinal Research Unit, Department of Ophthalmology, King's College Hospital, Denmark Hill, London, UK

Correspondence: S Sivaprasad, Laser and Retinal Research Unit, King's College Hospital, Denmark Hill, Normandy Building, London SE5 9RS, UK Tel: 0203299 4548; Fax: 02032993738. E-mail: senswathi@ aol.com

Received: 14 May 2009 Accepted in revised form: 18 September 2009 Published online: 6 November 2009

\begin{abstract}
Purpose This interventional, noncomparative case series assessed the outcome of intravitreal pan-anti-vascular endothelial growth factor (VEGF) agents in eyes with persistent or reactivated choroidal neovascularisation (CNV) following previous treatment with photodynamic therapy (PDT). Methods Baseline assessments including best-corrected visual acuity, fluorescein angiography (FFA), and optical coherent tomography (OCT) were performed.

Intravitreal ranibizumab and/ or bevacizumab were administered on a PRN basis, guided by changes in visual outcome and OCT findings. The follow-up period was at least 6 months. Results Twenty-five subjects with predominantly classic $\mathrm{CNV}$, previously treated with PDT (mean 1.84 PDT sessions) showed reactivation or persistent $\mathrm{CNV}$. The mean interval between PDT and intravitreal anti-VEGF treatment was $\mathbf{1 8 . 3 2}$ months (1-48 months); and patients received an average of 3.2 injections over a 6-month period. The mean change of visual acuity following PDT was -10.12 Early Treatment Diabetic Retinopathy Study (ETDRS) letters (54.36 \pm 15.79-44.24 \pm 17.32 letters). Following anti-VEGF therapy, the mean change in visual acuity at 3 and $\mathbf{6}$ months were $+\mathbf{1 . 7 6}$ and +0.72 , respectively. The proportion of subjects with stable vision (loss of $\leqslant 15$ letters) was $96 \%$ at 3 months and $88 \%$ at 6 months; the proportion of subjects who showed improved vision ( $\geqslant 15$ letters) was $8 \%$ at 3 months and $4 \%$ at 6 months.

Conclusions Anti-VEGF agents stabilised the visual outcomes of eyes previously treated with PDT. However, the proportion of patients who showed improved vision in this group
\end{abstract}

was smaller than the proportion reported in subjects with treatment-naive lesions. Eye (2010) 24, 1018-1023; doi:10.1038/eye.2009.266; published online 6 November 2009

Keywords: choroidal neovascularisation; bevacizumab; ranibizumab; photodynamic therapy; anti-VEGF

\section{Introduction}

Choroidal neovascularisation (CNV) secondary to neovascular age-related macular degeneration (AMD) cases are classified into angiographic subtypes depending on the proportion of the classic component into predominantly classic, minimally classic, and occult CNV. ${ }^{1}$ The aim of this treatment is to ablate or modulate $\mathrm{CNV}$ to minimise damage to adjacent structures. The Treatment of Age-Related Macular Degeneration with Photodynamic Therapy (TAP report 1) study reported in 1999 that $61 \%$ of subjects with predominantly classic CNV treated with Verteporfin (Visudyne: Novartis Pharmaceuticals Corp., East Hanover, NJ, USA) photodynamic therapy (PDT) lost fewer than 15 letters at 1 year, compared with $46 \%$ of subjects treated with placebo. ${ }^{2}$ Since then, PDT has been the treatment of choice for predominantly classic lesions.

Management of neovascular AMD is changing with advances in understanding its pathophysiology. ${ }^{3}$ Since the recent development of pharmacological agents that block vascular endothelial growth factor (VEGF) in CNV, wellconducted trials have shown that intravitreal ranibizumab, an anti-VEGF monoclonal antibody fragment that blocks all isoforms of VEGF-A, provides a much more effective 
alternative to the traditional techniques, which were at best able to stabilise (but rarely improve) visual acuity. ${ }^{4,5}$ The 12-month results of the 2-year phase III trial on monthly intravitreal ranibizumab therapy vs PDT for predominantly classic CNV showed that $96.4 \%$ of the $0.5 \mathrm{mg}$ ranibizumab group lost fewer than 15 letters, compared with $64.3 \%$ in the PDT arm (ANCHOR study). ${ }^{5}$ In this study, subjects in the PDT arm were allowed to cross over to ranibizumab therapy at 18 months or later when intravitreal ranibizumab became the standard care for all subtypes of CNV secondary to AMD. ${ }^{6}$

In daily practice, bevacizumab, a full-length monoclonal anti-VEGF antibody, became available for neovascular AMD before ranibizumab was authorised, ${ }^{7}$ and some subjects undergoing PDT opted for it, although it has not been licensed for intraocular use.

In this study, we report the outcomes of the use of ranibizumab and bevacizumab in consecutive subjects with AMD-related predominantly classic CNV previously treated with PDT; we compared the outcomes with those reported in patients who crossed over in the ANCHOR 2-year trial.

\section{Methods}

This study was approved by the local ethics committee; informed consent for the use of intravitreal ranibizumab and the associated risks, benefits, and treatment alternatives was obtained from all subjects. Intravitreal bevacizumab was used as a treatment option before ranibizumab became available in the NHS. Informed consent for the 'off-label' use of this drug was also obtained.

Consecutive subjects that presented with persistent or recurrent $\mathrm{CNV}$ following previous treatment with PDT for predominantly classic CNV were identified from a database maintained by the Laser and Retinal Research Unit, King's College hospital for anti-VEGF therapy. The database has been in operation since February 2007.

Inclusion criteria were: (1) age $\geqslant 50$ years; (2) clinical diagnosis of wet AMD; (3) predominantly classic CNV treated with PDT showing persistent or new activity; and (4) best-corrected visual acuity of 24-73 letters on the Early Treatment Diabetic Retinopathy Study (EDTRS) chart. ${ }^{8}$ Active CNV (persistent or recurrent) was defined as recent disease progression, as evidenced by a recent decline in vision, with new or persistent subretinal or intraretinal fluid on optical coherent tomography (OCT), an increase in lesion size $>10 \%$ and persistent leak as evidenced on fundus fluorescein angiogram (FFA), or new haemorrhage. Only one eye of each patient was included in the study.

Exclusion criteria included CNV from causes other than AMD (myopia, angioid streaks, choroidal rupture, chorio-retinal scarring, etc). Subjects with permanent structural damage at the fovea with limited visual potential (assessed by the treating physician) were excluded from the study. Patients were not excluded for pre-existing systemic health problems.

\section{Ocular examination}

All subjects underwent complete ophthalmic examination, including slit-lamp bio microscopic examination of the anterior segment, intraocular pressure measurement, and dilated fundoscopic examination of the posterior pole at every visit. FFA was carried out before the commencement of PDT and before initiation of anti-VEGF therapy; following this, it was performed at the discretion of the treating physician. OCT examinations were performed at every visit using a STRATUS OCT (Carl Zeiss, Meditec). The fast macular thickness map protocol was used for quantitative measures of the central macular thickness (CMT) and macular volume.

\section{Treatment options}

\section{Photodynamic therapy}

Subjects received standard fluence verteporfin PDT in accordance with the TAP protocol (verteporfin infusion over $10 \mathrm{~min}$ at a dose of $6 \mathrm{mg} / \mathrm{m}^{2}$; activating light applied $15 \mathrm{~min}$ after start of infusion at a wavelength of $689 \mathrm{~nm}$, light dose of $50 \mathrm{~J} / \mathrm{cm}$, and fluence of $600 \mathrm{~mW} / \mathrm{cm}^{2}$ ) at baseline. Subjects were followed up every 12 weeks, and FFA was repeated to assess activity. All subjects with reactivation of $\mathrm{CNV}$ were included in this case series. Subjects who were started on PDT at the time antiVEGF treatment became available were given the option to switch to anti-VEGF therapy if there was persistent disease activity without permanent structural damage to the fovea (as judged by the treating physician).

\section{Intravitreal ranibizumab/bevacizumab therapy}

After administration of topical anaesthesia, ranibizumab $0.5 \mathrm{mg} / 0.05 \mathrm{ml}$ or bevacizumab $1.25 \mathrm{mg} / 0.05 \mathrm{ml}$ was injected using a 30-G needle through a pars plana approach under aseptic conditions. Subjects selfadministered a topical antimicrobial agent (1\% ofloxacin ophthalmic solution) 4 times daily for 3 days after treatment. All subjects were followed up monthly. Re-injection of anti-VEGF agent was performed if visual acuity had deteriorated and (a) the OCT showed persistent or new fluid, (b) new haemorrhage was noted, (c) the lesion size increased by $\geqslant 10 \%$. 


\section{Outcome measures}

The primary efficacy end point was the proportion of subjects who lost fewer than 15 ETDRS letters at 6 months after initiation of anti-VEGF therapy. The change in visual outcome from the baseline (before the initiation of the first PDT session) was a secondary efficacy end point. Additional exploratory end points were the comparative outcomes of sequential therapy of bevacizumab followed by ranibizumab, vs ranibizumab monotherapy in subjects previously treated with PDT. The results were compared with the crossover group in the 2-year report of the ANCHOR study, in which subjects previously treated with PDT were allowed to cross over to the ranibizumab arm and were monitored for 6 months.

Multiple linear regression analysis was carried out to assess the factors that influenced the outcome. Factors assessed included visual acuity, CMT, macular volume before commencement of anti-VEGF therapy, number of PDT sessions, thickness of the subfoveal subretinal tissue at 6 months, presence of intraretinal cysts, and subretinal fluid, and macular fluid (defined as a combination of intraretinal fluid and subretinal fluid).

\section{Results}

\section{Patient demographics}

Twenty-five consecutive subjects with predominantly classic CNV who had been treated previously with PDT were included. The mean age was $79.84 \pm 5.76$ years (range 71-91 years). All subjects were Caucasian, with a comparable number of men (48\%) and women (52\%). There were four subjects with coexistent ocular pathology (two with non-proliferative diabetic retinopathy and two with primary open-angle glaucoma), and two subjects were pseudophakic.

\section{Baseline characteristics}

The mean total pre-PDT greatest linear diameter of the lesion was $1832 \mu \mathrm{m}$ (median $1850 \mu \mathrm{m}$; range 716-3000 $\mu \mathrm{m}$ ). The mean number of PDT sessions was 1.84 (range: $1-5$ sessions). No subjects received any combination treatments at baseline. Before treating with anti-VEGF regimen, one had intravitreal triamcinolone, two had sub tenons triamcinolone, and three had orbital floor triamcinolone as treatment for persistent activity of neovascular membrane. None had undergone laser treatment.

\section{Anti-VEGF therapy}

The interval between last PDT and start of anti-VEGF regime was 18.32 months (1-48 months). Eleven subjects were initiated on intravitreal bevacizumab and then switched to ranibizumab when the drug became available. This cohort received a mean of 1.8 intravitreal bevacizumab (1-4) and 0.4 ranibizumab (1-4). The remaining patients $(n=14)$ received ranibizumab monotherapy, with a mean of 3.6 injections (1-5). The mean number of anti-VEGF injections for the whole cohort was 3.2 in 6 months (1-5). Subanalysis of the pattern of injections revealed that $18(72 \%)$ received the first three injections on a monthly basis, followed by a PRN approach (3+ PRN approach), whereas seven (28\%) received PRN therapy throughout the treatment period.

\section{Changes in visual acuity}

Mean baseline visual acuity before PDT was $54.36 \pm 15.79$ ETDRS letters (35-85); at initiation of anti-VEGF treatment, mean visual acuity was $44.24 \pm 17.32$ ETDRS letters (13-80). There was a mean change in post-PDT visual acuity of -10.12 ETDRS letters during a mean follow-up of 18.32 months. Table 1 shows the mean

Table 1 Outcomes of anti-VEGF therapy in CNV previously treated with PDT

\begin{tabular}{|c|c|c|c|c|c|}
\hline & $\begin{array}{l}\text { Anti-VEGF onset } \\
\quad(\mathrm{n}=25)\end{array}$ & $\begin{array}{l}\text { Month } 1 \\
(\mathrm{n}=25)\end{array}$ & $\begin{array}{r}\text { Month } 2 \\
(\mathrm{n}=25)\end{array}$ & $\begin{array}{l}\text { Month } 3 \\
(\mathrm{n}=25)\end{array}$ & $\begin{array}{r}\text { Month } 6 \\
(\mathrm{n}=25)\end{array}$ \\
\hline Mean VA (ETDRS) & 44.24 & 46.32 & 46 & 46 & 44.96 \\
\hline Mean change in VA (ETDRS) since baseline (pre PDT) & -10.12 & -8.06 & -8.36 & -8.36 & -9.40 \\
\hline $\begin{array}{l}\text { Mean change in VA (ETDRS) since the start } \\
\text { of anti-VEGF regimen }\end{array}$ & Not applicable & +2.06 & +1.76 & +1.76 & +0.72 \\
\hline CMT & $328 \mu \mathrm{m}$ & $270 \mu \mathrm{m}$ & $283 \mu \mathrm{m}$ & $275 \mu \mathrm{m}$ & $281 \mu \mathrm{m}$ \\
\hline CFV & $7.47 \mathrm{~mm}^{3}$ & $6.92 \mathrm{~mm}^{3}$ & $7.35 \mathrm{~mm}^{3}$ & $7.72 \mathrm{~mm}^{3}$ & $8.17 \mathrm{~mm}^{3}$ \\
\hline Percentage losing $<15$ letters & NA & $100 \%$ & $100 \%$ & $96 \%$ & $88 \%$ \\
\hline Percent gaining $\geqslant 15$ letters & NA & none & none & $8 \%$ & $4 \%$ \\
\hline
\end{tabular}

CFV, central foveal volume; CMT, central macular thickness; CNV, choroidal neovascularisation; NA, not applicable; PDT, photodynamic therapy; VA, visual acuity; VEGF, vascular endothelial growth factor. 
change in visual acuity after commencement of antiVEGF therapy.

The mean change of visual acuity at 6 months in the group that had sequential therapy of bevacizumab followed by ranibizumab was $-1.82(-19$ to +11$)$, compared with +1.04 ETDRS letters $(-16$ to +17$)$ in the group treated with ranibizumab only. There was no statistical difference in the mean change in visual acuity in the $3+$ PRN group at 6 months $(+1.05(-19$ to +17$))$ compared with the PRN group, in whom mean change was -0.14 ETDRS letters $(-8$ to +7$)$. The visual acuity of two patients who developed retinal pigment epithelial tear dropped significantly ( -15 and -16 ETDRS letters, respectively), after initially gaining vision from antiVEGF treatment.

\section{Tomographic changes}

The mean CMT had decreased by $46.78 \mu \mathrm{m}(-237 \mu \mathrm{m}$ to $+97 \mu \mathrm{m}) 6$ months after initiation of anti-VEGF therapy, but there was no significant change in macular volume. The mean thickness of the subretinal tissue (using callipers) was $114.44 \mu \mathrm{m}$ at 6 months (range 54-222 $\mu \mathrm{m}$ ). At the 6-month follow-up, intraretinal fluid persisted in $28 \%$ of the patients, subretinal fluid persisted in $20 \%$, and macular fluid (intraretinal fluid plus subretinal fluid) persisted in $4 \%$ of the patients. Hence $56 \%$ had no fluid.

\section{Factors influencing outcomes}

Multiple logistic regression showed that baseline visual acuity $(P=0.04)$ and subfoveal subretinal tissue $(P=0.04)$ influenced the final visual outcome. Figure 1 shows the correlation between visual acuity change and subretinal tissue thickness $\left(r^{2}=0.5\right)$.

\section{Comparison of results to ANCHOR the crossover group}

Table 2 shows the comparison of our baseline characteristics and results with that of the ANCHOR crossover group $(n=50){ }^{6}$

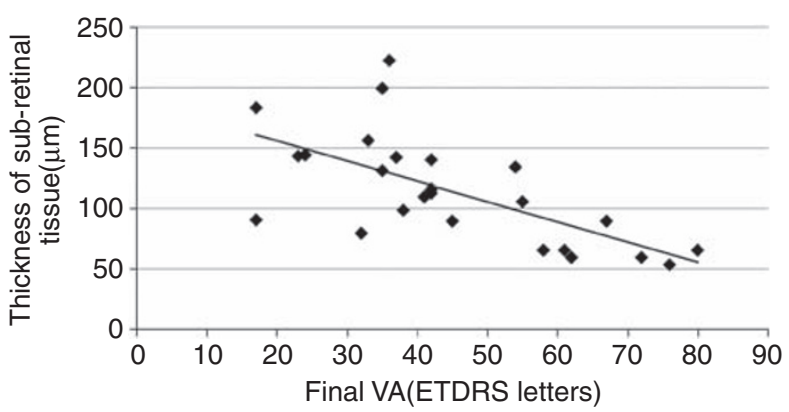

Figure 1 Correlation of final visual acuity (ETDRS letters) to thickness of subretinal tissue.
Figure 2 shows that there are no significant differences in the pattern of visual outcomes in the two groups following PDT as well as after anti-VEGF therapy. The gain in visual acuity is minimal with most subjects ending up with poorer vision compared with their baseline pre-PDT visual acuity. ${ }^{6}$

\section{Complications}

Two patients developed an extrafoveal RPE rip at the edge of the fibrotic lesion. There were no cases of increase in RPE atrophy, and no cases of endophthalmitis or uveitis.

\section{Discussion}

Several studies have shown the therapeutic efficiency and safety profile of intravitreal pan-VEGF-A inhibitors (ranibizumab and bevacizumab) in the management of CNV secondary to neovascular AMD. ${ }^{4-7}$ Most studies have opted for an OCT-guided regimen, based on the Prospective OCT Imaging of Patients with Neovascular AMD Treated with Intraocular Ranibizumab (PrONTO) study; these studies have shown stabilisation of visual acuity in more than $90 \%$ of the subjects and improvement in visual acuity in over $35 \%$ of the subjects. ${ }^{8,9}$ Studies assessing the outcome of a combination of anti-VEGF agents with PDT in various protocols have shown similar results. For example, FOCUS, a Phase I/II clinical study, investigated the safety and efficacy of ranibizumab in combination with PDT compared with PDT alone in subjects with predominantly classic wet AMD. ${ }^{10}$ Results at 12 months showed that $94.3 \%$ of those given $0.3 \mathrm{mg}$ of ranibizumab and $96.4 \%$ of those given $0.5 \mathrm{mg}$ lost fewer than 15 letters, as compared with $64.3 \%$ of those in the PDT group. Visual acuity improved by 15 letters or more in $35.7 \%$ of the $0.3 \mathrm{mg}$ group and $40.3 \%$ of the $0.5 \mathrm{mg}$ group, as compared with $5.6 \%$ of the PDT group. Mean visual acuity increased by 8.5 letters in the $0.3 \mathrm{mg}$ group and 11.3 letters in the $0.5 \mathrm{mg}$ group, and it decreased by 9.5 letters in the PDT group.

In this case series, we evaluated outcomes of treatment with ranibizumab and bevacizumab in $\mathrm{CNV}$ that had been previously treated with PDT. We found that $88 \%$ of patients lost fewer than 15 letters, and visual acuity improved by 15 letters or more in $4 \%$ at 6 months. The mean visual acuity increased by +0.72 letters. These results show that the visual outcomes of this group are worse than that in treatment-naive lesions treated with anti-VEGF monotherapy or a combination of anti-VEGF and PDT.

Our results are similar to that obtained at 6 months in the 50 subjects who crossed over to the ranibizumab arm from the PDT arm in the ANCHOR study. ${ }^{6}$ 
Table 2 Comparison of outcomes of present series vs the ANCHOR crossover group

\begin{tabular}{|c|c|c|}
\hline & $\begin{array}{l}\text { Anchor crossover group }(\mathrm{n}=50) \\
\text { Crossover to Ranibizumab at } 18 \text { months }\end{array}$ & Present series $(\mathrm{n}=25)$ \\
\hline Age mean (SD) & $76.3(7.9)$ & $79.84(5.76)$ \\
\hline Mean interval between PDT and anti-VEGF regimen & 18 months & 18.32 months \\
\hline Baseline mean VA & 43.9 & 54.36 \\
\hline $\mathrm{VA} \geqslant 45$ & 26 & 20 \\
\hline $\mathrm{VA}<45$ & 24 & 5 \\
\hline \multicolumn{3}{|l|}{ CNV lesion subtype: } \\
\hline Predominantly classic $n(\%)$ & $50(100 \%)$ & $25(100 \%)$ \\
\hline No. of PDT sessions & 3.8 & 1.84 \\
\hline Mean VA at the onset of anti-VEGF regimen & 38.5 & 44.24 \\
\hline Follow-up duration & 6 months & 6 months \\
\hline Mean number of injections & 3.3 & 3.2 \\
\hline Frequency of injections & monthly & OCT-guided \\
\hline Dosage of anti-VEGF (in $0.05 \mathrm{ml}$ ) & $0.3 \mathrm{mg}$ ranibizumab & $\begin{array}{c}0.5 \mathrm{mg} \text { ranibizumab } / 1.25 \mathrm{mg} \\
\text { bevacizumab }\end{array}$ \\
\hline Mean VA 6 months after starting anti-VEGF regimen & 38.2 & 44.96 \\
\hline Mean VA gain since anti-VEGF regimen & +0.2 & +0.72 \\
\hline Mean change in VA since baseline (pre-PDT) & -5.7 ETDRS letters & -9.4 ETDRS letters \\
\hline Change in mean CMT & NA & $-47.36 \mu \mathrm{m}$ \\
\hline
\end{tabular}

ANCHOR, Anti-VEGF Antibody for the Treatment of Predominantly Classic Choroidal Neovascularisation in AMD; CMT, central macular thickness; CNV, choroidal neovascularisation; ETDRS, Early Treatment Diabetic Retinopathy Study; OCT, optical coherent tomography; PDT, photodynamic therapy; SD, standard deviation; VA, visual acuity; VEGF, vascular endothelial growth factor.

The ANCHOR crossover group received a dose of $0.3 \mathrm{mg} / 0.5 \mathrm{ml}$ every month, whereas in our study, the subjects received $0.5 \mathrm{mg} / 0.05 \mathrm{ml}$ in an OCT-guided regimen. In addition, our cohort also had better baseline mean vision (pre-PDT), better mean visual acuity at the start of anti-VEGF therapy and had less number of PDT sessions (Table 2). The reasons for better baseline mean visual acuity in our series could be attributed to the fact that $10 \%$ of the crossover patients in the ANCHOR study had recurrent $\mathrm{CNV}$ after previous laser treatment, which could have affected the overall baseline visual acuity whereas the patients in our series were treatment-naive patients before photodynamic therapy.

The differences in outcome of post-PDT cases compared with the original ANCHOR study may be related to the PDT-induced collateral damage to choriocapillaries, RPE, and retina. ${ }^{11}$ Lesions treated with PDT usually result in retinal pigment epithelial atrophy and/or subretinal fibrosis. ${ }^{12}$ We analysed several factors that might influence final visual outcome including baseline visual acuity (pre-PDT), pre-anti-VEGF visual acuity, size of lesion, number of PDT treatments, thickness of subfoveal subretinal tissue, macular volume, central retinal thickness, area of angiographic leakage, number of anti-VEGF agents, and the number of treatments with anti-VEGF agents. Of all these factors, only baseline visual acuity (pre-PDT) and the thickness of subfoveal subretinal tissue influenced the final visual outcome. Recent OCT classification of morphological appearance of $\mathrm{CNV}$ have indicated that the subretinal hyper-reflective band corresponds to fibrosis. ${ }^{13}$

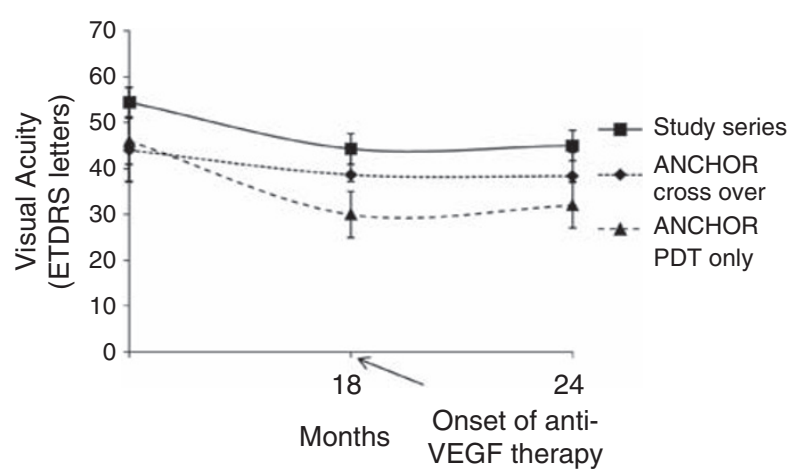

Figure 2 Comparison of VA outcome with ANCHOR cross over and PDT only patients.

Therefore, the thicker the subfoveal subretinal tissue, the worse the final visual outcome.

It is important to differentiate this study from studies that have assessed the outcome of combining anti-VEGF agents with PDT in various protocols. ${ }^{10,14}$ The combination treatments have shown better visual outcomes. In PDT, micro vascular occlusion induces inflammation and hypoxia and the expression of VEGF, which could lead to CNV persistence and recurrence. For that reason, synergistic inhibition of VEGF with PDT provides a multipronged attack on the $\mathrm{CNV} .{ }^{15,16}$ However, this study shows that, unlike the combination therapy, sequential therapy of PDT followed by anti-VEGF at a later date does not provide a similar synergistic effect. This may be because anti-VEGF agents can only modulate the $\mathrm{CNV}$ by decreasing the 
PDT-induced VEGF drive and have minimal or no effect on the atrophic and fibrotic changes induced by PDT in the long-term.

This study showed that it is safe to administer antiVEGF agents into PDT-treated eyes. There were two cases of RPE rip at the extrafoveal edge of the fibrosis. This may be a response to the tangential force on the fibrosis caused by sudden decrease in fluid after antiVEGF therapy. None of the subjects showed an increase in RPE atrophy.

This study provides a real-life experience of using antiVEGF agents in eyes previously treated with PDT. Our study has a few weaknesses. The most important limitation was the lack of a control group treated with PDT alone. However, as the ANCHOR study clearly showed the superior outcomes of intravitreal ranibizumab over PDT, it is not ethically appropriate to continue treating subjects with PDT. ${ }^{5}$ Second, our 6-month follow-up period is relatively short in the context of the natural history of the disease; long-term treatment efficacy remains to be determined. Third, the optimal dosage, number, and frequency of intravitreal ranibizumab/bevacizumab injections in eyes previously treated with PDT remain uncertain. Our study showed that the subjects who received the first three injections on a fixed monthly schedule did not fare better than the group treated on OCT-guided PRN basis from the first visit. We also noted no difference in outcome between the group treated with ranibizumab alone and the group treated with a combination of bevacizumab and ranibizumab. It may be argued that the small sample size may have skewed the results in the subgroup analysis. However, our study outcomes compared very well with the crossover group of a well designed randomised controlled trial (ANCHOR 2-year study). We conclude that activation of $\mathrm{CNV}$ in eyes previously treated with PDT can be treated safely with an OCT-guided regimen of intravitreal anti-VEGF agents. However, patients should be informed that the aim of anti-VEGF therapy is stabilisation of vision, and the likelihood of visual improvement is limited.

\section{Conflict of interest}

The authors declare no conflict of interest

\section{References}

1 Macular Photocoagulation Study Group. Subfoveal neovascular lesions in age-related macular degeneration. Guidelines for evaluation and treatment in the Macular Photocoagulation Study. Arch Ophthalmol 1991; 109: 1242-1257.

2 Treatment of Age-related Macular Degeneration with Photodynamic Therapy (TAP) Study Group. Photodynamic therapy of subfoveal choroidal neovascularization in age-related macular degeneration with verteporfin. Arch Ophthalmol 1999; 117: 1329-1345.

3 Adamis AP, Shima DT. The role of vascular endothelial growth factor in ocular health and disease. Retina 2005; 25(2): 111-118

4 Rosenfeld PJ, Brown DM, Heier JS, Boyer DS, Kaiser PK, Chung CY et al. MARINA Study Group. Ranibizumab for neovascular age-related macular degeneration. $N$ Engl J Med 2006; 355: 1419-1431.

5 Brown DM, Kaiser PK, Michels M, Heier JS, Sy JP, Ianchulev $\mathrm{T}$ et al. ANCHOR Study Group. Ranibizumab versus verteporfin for neovascular age-related macular degeneration. N Engl J Med 2006; 355: 1432-1444.

6 Brown DM, Michels M, Kaiser PK, Heier JS, Sy JP, Ianchulev T. ANCHOR Study Group. Ranibizumab versus verteporfin photodynamic therapy for neovascular age-related macular degeneration: two-year results of the ANCHOR study. Ophthalmology 2009; 116(1): 57-65.e5.

7 Spaide RF, Laud K, Fine HF, Klancnik Jr JM, Meyerle CB, Yannuzzi LA et al. Intravitreal bevacizumab treatment of choroidal neovascularization secondary to age-related macular degeneration. Retina 2006; 26: 383-390.

8 Fung AE, Lalwani GA, Rosenfeld PJ, Dubovy SR, Michels S, Feuer $\mathrm{W}$ et al. An optical coherence tomography-guided, variable dosing regimen with intravitreal ranibizumab (Lucentis) for neovascular age-related macular degeneration. Am J Ophthalmol 2007; 143(4): 566-583.

9 Arias L, Caminal JM, Casas L, Masuet C, Badia MB, Rubio $\mathrm{M}$ et al. A study comparing two protocols of treatment with intravitreal bevacizumab (Avastin) for neovascular agerelated macular degeneration. Br J Ophthalmol 2008; 92(12): 1636-1641.

10 Antoszyk AN, Tuomi L, Chung CY, Singh A, FOCUS Study Group. Ranibizumab combined with verteporfin photodynamic therapy in neovascular age-related macular degeneration (FOCUS): year 2 results. Am J Ophthalmol 2008; 145(5): 862-874.

11 Michels S, Schmidt-Erfurth U. Sequence of early vascular events after photodynamic therapy. Invest Ophthalmol Vis Sci 2003; 44(5): 2147-2154.

12 Sivaprasad S, Saleh GM, Jackson H. Does lesion size determine the success rate of photodynamic therapy for age-related macular degeneration? Eye 2006; 20(1): 43-45.

13 Patel PJ, Browning AC, Chen FK, Da Cruz L, Tufail A. Interobserver agreement for the detection of optical coherence tomography features of neovascular age-related macular degeneration. Invest Ophthalmol Vis Sci 2009, e-pub ahead of print 24 June 2009.

14 Kumar A, Gopalakrishnan K, Sinha S. Combination photodynamic therapy and intravitreal ranibizumab in neovascular age-related macular degeneration in a North Indian population: a pilot study. Retina 2008; 28(9): 1296-1301.

15 Zuluaga MF, Mailhos C, Robinson G, Shima DT, Gurny R, Lange N. Synergies of VEGF inhibition and photodynamic therapy in the treatment of age-related macular degeneration. Invest Ophthalmol Vis Sci 2007; 48(4): 1767-1772.

16 Ju M, Mailhos C, Bradley J, Dowie T, Ganley M, Cook G et al. Simultaneous but not prior inhibition of VEGF165 enhances the efficacy of photodynamic therapy in multiple models of ocular neovascularization. Invest Ophthalmol Vis Sci 2008; 49(2): 662-670. 